Le NGS (Next-Generation Sequencing, Séquençage de Nouvelle Génération) a conduit depuis son avènement en 2005 à une révolution dans la dimension des analyses génétiques, par un changement majeur d'échelle des capacités de séquençage, avec de nombreuses applications initialement dans le domaine de la recherche, puis largement dans le domaine du diagnostic des maladies monogéniques hétérogènes [1]. Dans le cadre du second Plan National Maladies Rares (PNMR2 ; 2011-2014), les Laboratoires de Diagnostic Génétique des Centres Hospitaliers français ont été équipés progressivement en plateformes de NGS. L'implémentation du NGS dans les laboratoires hospitaliers français a permis l'intégration de ces analyses dans la démarche diagnostique pour de nombreuses pathologies génétiquement hétérogènes, dont les myopathies. Les analyses classiques par séquençage Sanger ont alors été progressivement remplacées par des approches NGS, majoritairement de type «panel de gènes » [1-3]. Néanmoins, en raison d'un manque initial de concertation des laboratoires sur le plan national, l'utilisation en routine diagnostique du NGS a rapidement été confrontée à des problèmes d'hétérogénéité à différents niveaux, notamment en termes de technologies utilisées (type de séquenceur), de listes de gènes analysées, de classification des variants identifiés, et d'intégration du NGS dans la démarche diagnostique. Ceci a conduit à une grande disparité dans l'offre diagnostique actuelle, complexifiant la visibilité pour les cliniciens prescripteurs («Quelle prescription d'analyses génétiques pour quelle indication, et où puis-je envoyer l'échantillon de mon patient atteint de myopathie?»).

Cette problématique a été abordée sur le plan national depuis 2015 dans le cadre de différentes Filières de Soin Maladies Rares, et en lien avec l'Association Nationale de Praticiens en Génétique Moléculaire (ANPGM), avec la constitution de commissions ou groupes de travail spécifiques. Ceci a permis d'initier des travaux d'homogénéisation nationale des analyses par NGS (Figure 1). Dans le domaine des myopathies, le Sous-groupe Génétique Moléculaire de la Commission Outils diagnostiques de notre filière FILNEMUS a engagé différentes actions en ce sens, qui ont été présentées lors de la journée NGS-FILNEMUS en mars 20172017 (vidéos des présentations disponibles sur le site www.filnemus.fr). Par

Vignette (Photo $\odot$ Pixabay - Creative Commons).

\section{Vers une \\ homogénéisation \\ nationale des \\ analyses par NGS dans la démarche diagnostique pour les myopathies}

\author{
Martin Krahn ${ }^{1,2}$, Mathieu Cerino ${ }^{1,2}$, Emmanuelle \\ Campana-Salort ${ }^{1,3}$, Mireille Cossée ${ }^{4,5}$
}

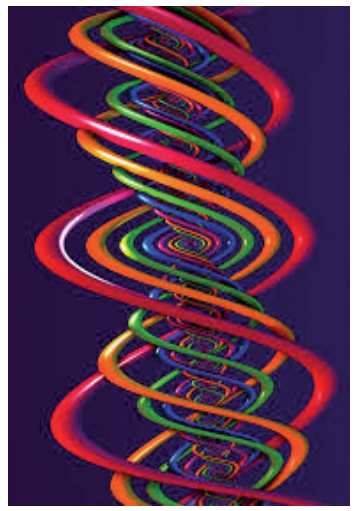

${ }^{1}$ Aix Marseille Université, Inserm UMR_S 910, GMGF, 13385, Marseille, France.

${ }^{2}$ APHM, Département de Génétique Médicale, Hôpital

Timone Enfants, 13385, Marseille, France.

${ }^{3}$ APHM, Hôpital Timone, Centre de référence des maladies neuromusculaires et de la SLA, Marseille, France.

${ }^{4} \mathrm{CHRU}$ Montpellier, Laboratoire de Génétique moléculaire,

ailleurs, il est apparu rapidement que Montpellier, France.

${ }^{5}$ Université Montpellier,

Laboratoire de Génétique de maladies rares, Montpellier, France.

martin.krahn@univ-amu.fr mireille.cossee@inserm.fr ridisciplinaire (RCP) dédiée.

Nous avons souhaité présenter ici de manière synthétique, sous la forme d'un tableau détaillé (Tableau I), un point d'étape clair récapitulant les différentes actions, finalisées, en cours ou prévues, depuis 2015 pour une homogénéisation nationale des analyses par séquençage de nouvelle génération dans la démarche diagnostique pour les myopathies. Ces actions seront activement poursuivies en coordination avec les futures plateformes nationales de séquençage à très haut débit (Plan France Médecine Génomique 2025). $\diamond$

Towards a national standardisation of NGS studies in the diagnosis of myopathies

\section{REMERCIEMENTS}

Les auteurs remercient vivement leurs collègues cliniciens et de laboratoire, ainsi que les membres du Sous-groupe Génétique Moléculaire de la Commission Outils Diagnostiques de FILNEMUS et des différents groupes de travail de l'ANPGM, pour leur implication dans l'homogénéisation nationale des analyses par NGS. 

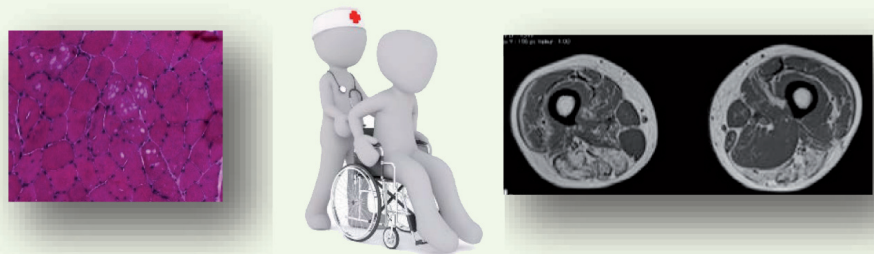

Document de recueil synthétique des données cliniques et paracliniques (en cours)

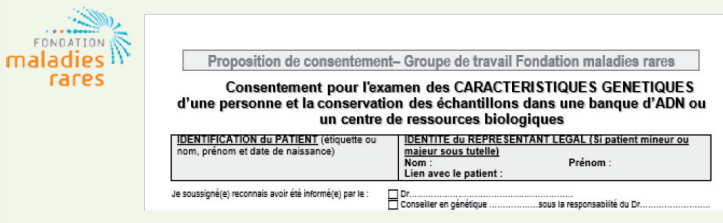

Homogénéisation du consentement éclairé

Homogénéisation des panels de gènes analysés par NGS

Arbres décisionnels incluant les panels de gènes (en cours)
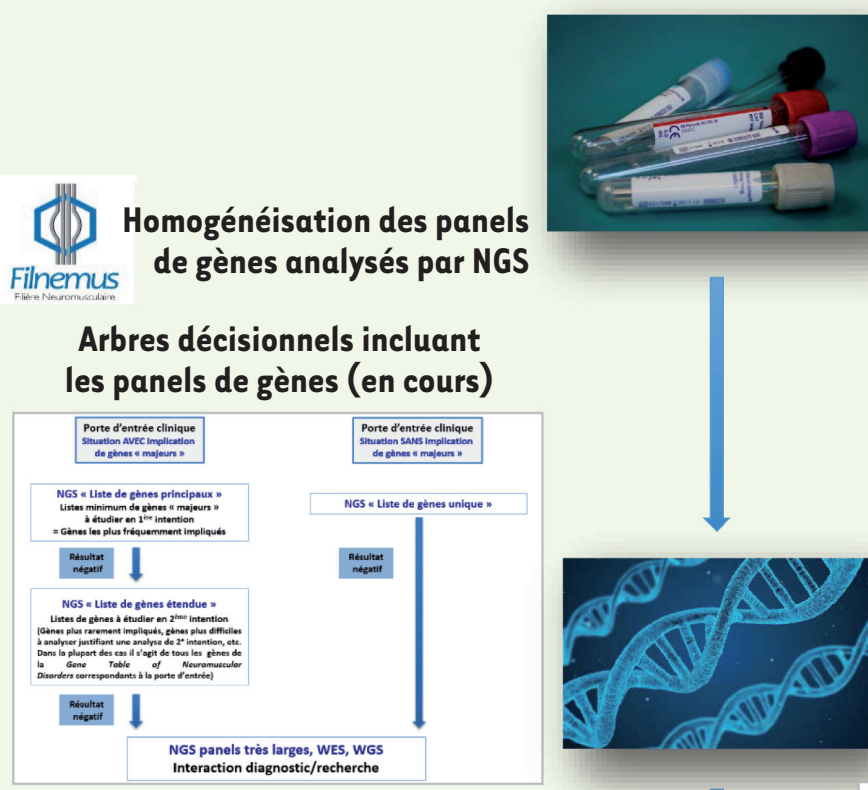

Inventaire des laboratoires
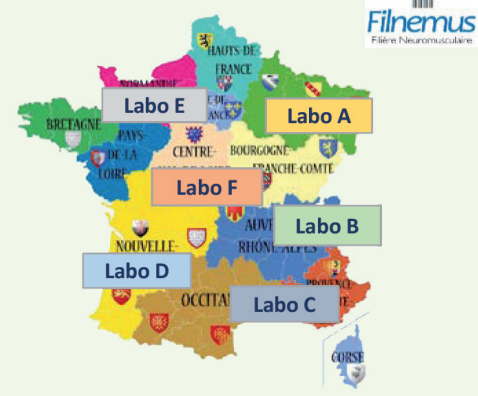

Filnemus (anges, wes, WGs

Systématisation des interactions avec laboratoires de recherche pour la validation fonctionnelle de pathogénicité des variants (prévu)

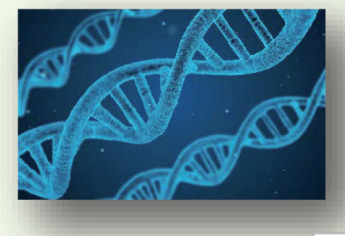

Homogénéisation de la classification des variants (en cours)

Figure 1. Vue d'ensemble des actions d'homogénéisation nationale des analyses par NGS dans la démarche diagnostique pour les myopathies. Le descriptif détaillé des actions nationales FILNEMUS et hors FILNEMUS réalisées depuis 2015 ou prévues est présenté dans le tableau. RCP : Réunions de Concertation Pluridisciplinaires; NGS : Séquençage de Nouvelle Génération. 


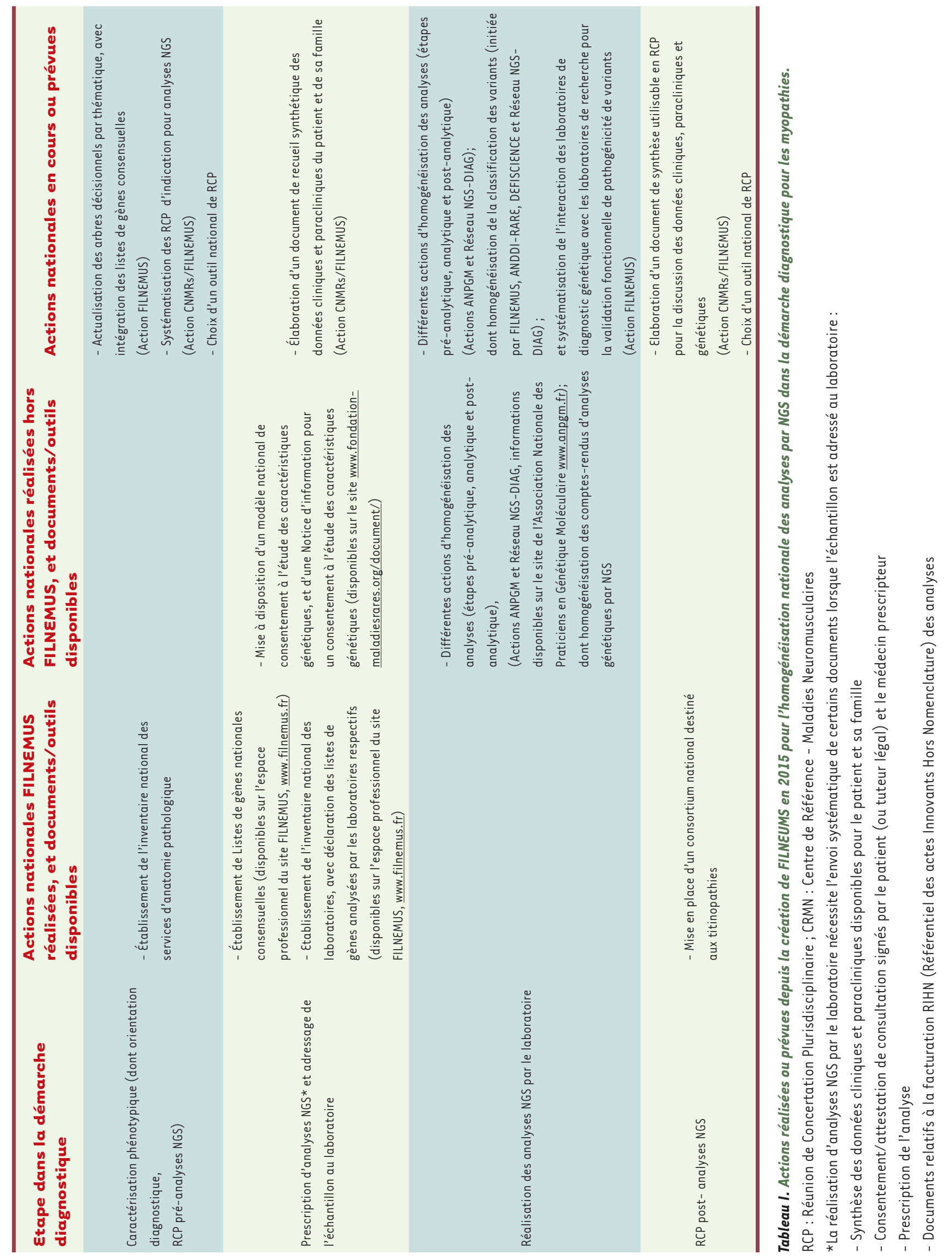




\section{LIENS D'INTÉRÊT}

Les auteurs déclarent n'avoir aucun lien d'intérêt concernant les don- nées publiées dans cet article.

\section{RÉFÉRENCES}

1. Krahn M, Lévy N, Bartoli M. Le séquençage de nouvelle génération appliqué au diagnostic de maladies monogéniques hétérogènes : notions essentielles pour le dialogue entre cliniciens et généticiens. Cah Myol 2016; 13 : 31-3.

2. Krahn $M$, Arveiler $B$. Le séquençage de nouvelle génération : principe, applications en diagnostic et perspectives. In : Livre national d'enseignement-Génétique Médicale-DFGSM2/3. Issy-lesMoulineaux : Elsevier-Masson, 2016.
3. Gorokhova S, Biancalana V, Lévy N, et al. Clinical massively parallel sequencing for the diagnosis of myopathies. Rev Neurol $2015 ; 171$ : 558-71.
TIRÉS À PART

M. Krahn

\section{La cartographie de la filière FILNEMUS évolue...}

\section{d'après l'arrêté ministériel du 15 septembre 2017}
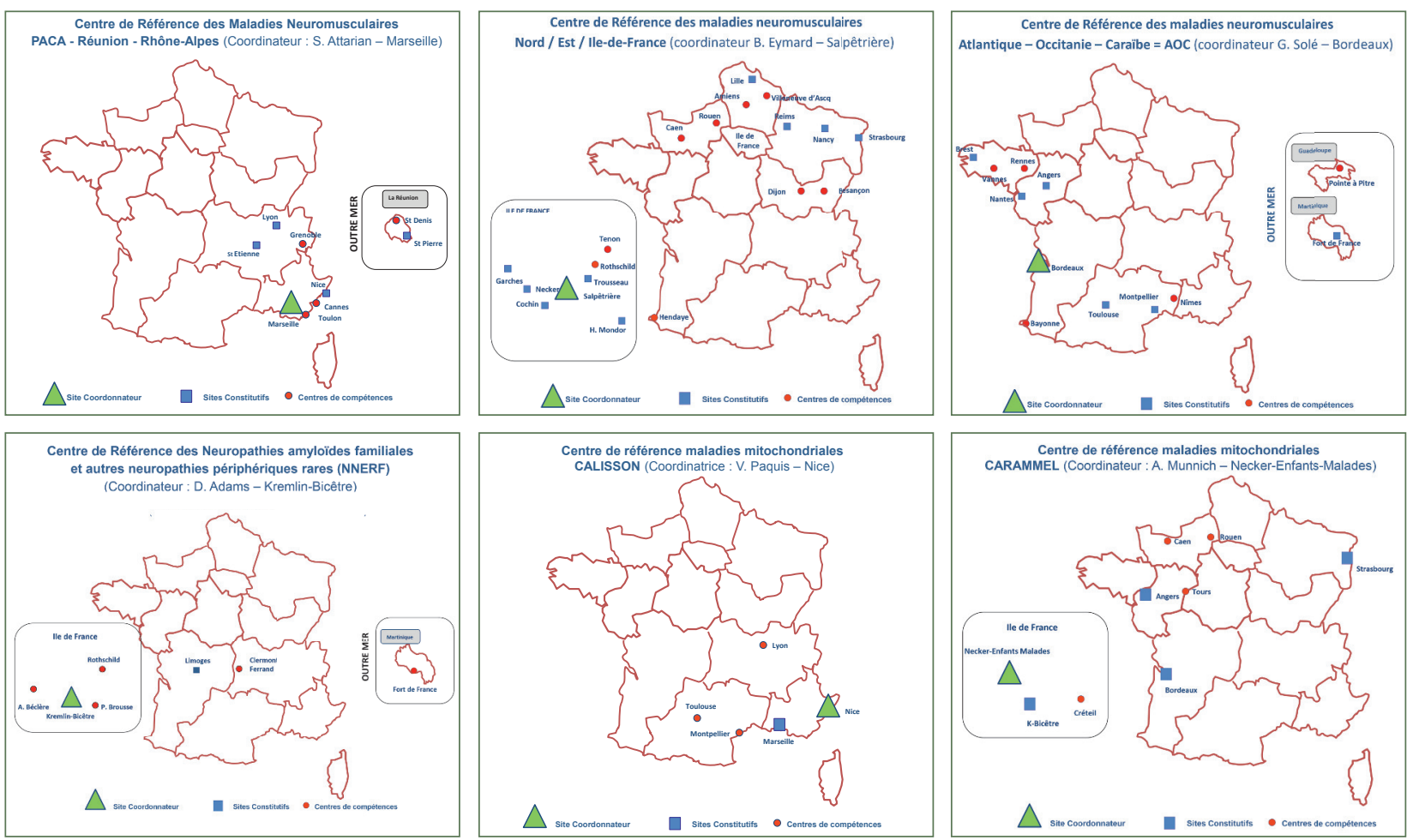

FILNEMUS est une des 23 Filières de Santé Maladies Rares (FSMR) retenues par le Ministère dans le cadre du second plan national maladies rares 2011-2016. Les affections relevant de la filière FILNEMUS incluent les maladies du muscle (myopathies), les maladies de la jonction neuromusculaire, les maladies rares du nerf périphérique et les amyotrophies spinales infantiles. À ce jour, on compte en France entre 40000 et 50000 personnes atteintes de pathologie neuromusculaire.

FILNEMUS rassemble les centres de références et les centres de compétence neuromusculaires et intègre également les laboratoires de diagnostic, l'ensemble des acteurs de la prise en charge pluridisciplinaire, les chercheurs travaillant sur la thématique neuromusculaire, les associations de patients, les acteurs du domaine médico-social ainsi que les sociétés savantes concernées par les maladies neuromusculaires.

FILNEMUS a pour objectifs d'améliorer le diagnostic et la prise en charge des patients atteints de maladies neuromusculaires et de favoriser les interactions entre les différents acteurs au service du patient.

Le site internet de FILNEMUS www.filnemus.fr est un outil d'informations et de communication à destination des professionnels concernés par la Filière ainsi que pour les patients atteints de maladies neuromusculaires et leur famille. 